\title{
(I) OPEN ACCESS \\ Do we need a subspecialty curriculum for training in pancreaticobiliary medicine in the UK?
}

\author{
Andrew Hart, ${ }^{1}$ Gavin Johnson, ${ }^{2}$ Matthew Huggett ${ }^{3}$
}

\begin{abstract}
${ }^{1}$ Norwich Medical School University of East Anglia, Norwich, UK

${ }^{2}$ University College Hospital, London, UK

${ }^{3}$ Department of

Gastroenterology, St James's University Hospital, Leeds, UK
\end{abstract}

\section{Correspondence to}

Dr Andrew Hart, Norwich Medical School, University of East Anglia, Norwich, NR4 7TJ, UK:

a.hart@uea.ac.uk

Received 16 July 2014 Revised 13 October 2014 Accepted 13 October 2014 Published Online First

4 November 2014

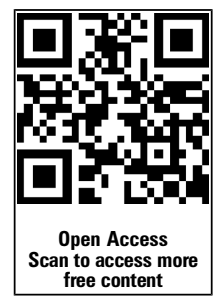

CrossMark

To cite: Hart A, Johnson G, Huggett M. Frontline Gastroenterology 2015:6:175-177.

\section{INTRODUCTION}

Pancreaticobiliary diseases, including acute and chronic pancreatitis and pancreatic cancer, are common, although there is currently no training curriculum for this subspecialty within medical gastroenterology. There is a need for medical gastroenterologists experienced in the management of these complex patients, which increasingly involves a multidisciplinary approach. For both acute and chronic pancreatitis, an aetiology should be sought and surveillance conducted for the development of complications, such as exocrine and endocrine failure, nutritional deficiencies and osteoporosis. Furthermore, some patients may require long-term support with smoking and alcohol cessation. Pancreatic cancer and cholangiocarcinoma have a poor prognosis that has not improved in recent years. Although physicians are involved in the initial diagnostic process, there are now evolving areas where they can contribute to the subsequent management. An increasing workload is the assessment and treatment of patients with cystic pancreatic lesions, detected incidentally on crosssectional imaging. Additionally, the management of biliary disorders, such as complex strictures and Sphincter of Oddi dysfunction, is equally challenging and requires close working between hepaticopancreaticobiliary (HPB) physicians, radiologists, surgeons and hepatologists.

A career in pancreaticobiliary medicine may appeal to doctors in training. There are rapidly developing diagnostic and therapeutic procedures for patients with pancreatic disease, emerging new clinical problems in the acute and long-term settings, together with a need for a personalised management approach. More physicians with such an interest will be required as the incidence of many pancreaticobiliary disorders is expected to rise as their aetiology is related to obesity and higher alcohol consumption. This paper discusses the potential development and components of a training programme in medical pancreatology, and the authors would welcome feedback on its suggestions.

\section{CURRICULUM CONSIDERATIONS}

The Joint Royal Colleges of Physicians Training Board (JRCPTB) Gastroenterology and Hepatology curriculum 2010 defines the process of training and the required competencies for the award of a certificate of specialty training in gastroenterology. ${ }^{1}$ This curriculum documents the further competencies required to achieve accreditation in the subspecialties of hepatology, inflammatory bowel disease and advanced nutrition. There is also the subspecialty of advanced endoscopy, which is specific to the technical aspects of endoscopic retrograde cholangiographic pancreatography (ERCP) and endoscopic ultrasound (EUS), but not to the management of patients with complex pancreatic and biliary disorders. The curriculum does define basic competencies in pancreaticobiliary medicine (gallbladder, acute pancreatitis, chronic pancreatitis and pancreatic tumours) required for all trainees, but there are many specific and detailed areas that are omitted. A UK specialised training in pancreaticobiliary disease could be enhanced by the means of a subspecialty curriculum for advanced training in this area, rather than just the technical aspects of specific endoscopic procedures (ERCP and EUS).

\section{COMPONENTS OF A CURRICULUM}

A curriculum in pancreaticobiliary medicine should include three basic 
components: (i) clinical outpatient diagnosis and management of pancreaticobiliary diseases; (ii) diagnostic and therapeutic radiological and endoscopic procedures and (iii) delivery of multidisciplinary meetings for both benign and malignant disorders. These three areas are now discussed.

i. In the outpatient clinic, the pancreaticobiliary physician provides a specialist service for the diagnosis and management of the relevant conditions, a service that currently does not exist in many hospitals. There is an emerging range of such disorders requiring specialist consideration and competencies, including

- investigation of the aetiology of acute and chronic pancreatitis

- long-term management of chronic pancreatitis

- assessment of patients with incidentally detected pancreatic cysts

- diagnosis and coordination of care of pancreatic cancer and cholangiocarcinoma

- management of emerging fields such as IgG4-related disease

- diagnosis of patients with biliary strictures

- assessment of patients with functional biliary disorders and indications for further investigation and treatment (Sphincter of Oddi dysfunction).

In both acute and chronic pancreatitis, techniques for investigating the underlying cause are advancing with the development of, for example, EUS for detecting microlithiasis. Patients with chronic pancreatitis benefit from long-term follow-up by ensuring an adequate nutritional status is maintained with appropriate pancreatic enzyme replacement and dietary supplements; monitoring for the development of diabetes, pancreatic cancer and osteoporosis; and effective pain control and psychological support for those with alcohol and smoking-related disease and who require cessation support. Patients with pancreatic cancer are often diagnosed by medical gastroenterologists, but their care is continued by oncologists, surgeons or palliative care physicians. Physicians can provide an important role by assessing the need for endoscopic stenting, pain control, nutritional requirements, monitoring for diabetes and depression, providing information and coordinating support and assessing whether there is a family history of this cancer to determine suitability for surveillance of relatives.

An evolving role for the pancreaticobiliary physician is in the management of patients with incidentally detected pancreatic cysts, some of which are premalignant (intraductal papillary mucinous neoplasms and mucinous cystic neoplasms). Incidental pancreatic cysts are reported in approximately $2 \%$ of abdominal CT scans requested for the investigation of abdominal or pelvic pain (not related to the cyst), or in the surveillance of patients following treatment of other cancers. $^{2}$ The characterisation and management of such cysts involves an awareness of the diagnostic modalities, as well as an assessment of the risks and benefits of surgical intervention, based on the degree of potential risk of malignant transformation of the cyst, and the consideration of any comorbidities and treatment wishes. Specialist medical pancreatology training is required to deliver effective care in this evolving area.

ii A second role for the pancreaticobiliary physician is to provide the emerging range of radiological, endoscopic diagnostic and therapeutic services. These competencies are already described as part of the module in advanced endoscopy; however, we would argue that they would be more relevant and could be expanded in a new pancreaticobiliary medicine curriculum. The competencies include EUS and diagnostic fine needle aspiration (FNA), EUS coeliac plexus neurolysis (EUS-CPN) and ERCP. Previously obtaining cytological or histological diagnosis of pancreatic cancer was difficult due to the relative inaccessibility of the pancreas via ultrasound or CT-guided approaches. This has been transformed with the established use of linear EUS and FNA/biopsy for sampling of both solid and cystic pancreatic lesions. ${ }^{3}$ EUS-CPN can deliver effective analgesia, via injection, of neurolytic agents into the coeliac plexus. ${ }^{4}$ For patients with chronic pancreatitis, ERCP can allow removal of pancreatic ductal stones (with or without extracorporeal shock wave lithotripsy) and treatment of duct strictures in selected patients in whom surgery may be inappropriate. $^{5}$ Minimally invasive EUS-guided techniques are developing and may in time become the standard of care for the management of peripancreatic fluid collections. ${ }^{6}$ Ideally, the junior physician would become competent in the basic therapeutic skills of interventional ERCP and of diagnostic EUS, which would then develop on their appointment to a consultant position. Trainees with a particular aptitude for interventional procedures may be taught others including cholangioscopy, intraductal ultrasound, complex pancreatic endotherapy (including extracorporal shock wave lithotripsy) and therapeutic EUS.

iii Training for pancreaticobiliary physicians should include active participation in multidisciplinary team (MDT) meetings for both benign and malignant pancreaticobiliary disease. Tertiary HPB cancer centres have forums for discussing patients with cancer, but few have equivalent pathways for patients with chronic pancreatitis, who have equally difficult clinical problems. Chairing an MDT involves an overview of the diagnostic procedures and management of pancreatic conditions, as well as organisational skills in assembling the necessary professionals and auditing the clinical outcomes. With the dismal prognosis of pancreaticobiliary cancers, physicians could also coordinate recruitment of patients into clinical trials, and knowledge of such local, national and international studies is imperative.

The training of junior physicians with an interest in pancreaticobiliary medicine should occur in units that provide a complete and comprehensive range of 
services for patients with pancreaticobiliary disorders. These services as described above are outpatient clinics, endoscopic and radiological procedures and active involvement in MDT meetings with physicians who work closely with HPB surgical units, specialist oncologists and histopathologists. Although at present such a range of services are available in only a few departments in the UK, an aim of the new curriculum would be to train gastroenterologists who would start developing such facilities elsewhere. The increase in the number of fully trained consultants would inevitably make training more widely available in the future. A relevant template for such a development could be based on the recent British Society of Gastroenterology (BSG) publication on the provision of ERCP services that documents several criteria including quality measures, minimum number of procedures performed and mentorship for new consultants. ${ }^{7}$ The number of hospitals required to deliver such a training curriculum in pancreaticobiliary medicine is currently unknown. However, with the expected rise in the requirement for HPB services, and the increasing range of interventional services, this requirement should be formally assessed if national organisations for training in gastroenterology accept the need for this new curriculum.

\section{FUTURE DEVELOPMENTS}

This paper has suggested reasons for justifying the development of an advanced training subspecialty curriculum in pancreaticobiliary medicine. The authors would welcome comments on our proposal from all health professionals involved in this area, including specialty registrars, as to whether this would be an attractive and rewarding career pathway. This training should perhaps be a mandated part of that of any gastroenterologist looking to develop a career in ERCP and pancreaticobiliary EUS. If there is a consensus for this proposal, we would work with the JRCPTB Specialist Advisory Committee and the BSG with the ultimate aim of improving the care and quality of life for patients with these aggressive and debilitating conditions.

Contributors All authors were involved in the conception and writing of this opinion piece.

Competing interests None.

Provenance and peer review Not commissioned; externally peer reviewed.

Open Access This is an Open Access article distributed in accordance with the Creative Commons Attribution Non Commercial (CC BY-NC 4.0) license, which permits others to distribute, remix, adapt, build upon this work non-

commercially, and license their derivative works on different terms, provided the original work is properly cited and the use is non-commercial. See: http://creativecommons.org/licenses/by$\mathrm{nc} / 4.0 /$

\section{REFERENCES}

1 http://www.jrcptb.org.uk/trainingandcert/ST3-SpR/Documents/ 2010\%20(AUC)\%20Gastroenterology\%20curriculum (amendments\%202013).pdf

2 Laffan TA, Horton KM, Klein AP, et al. Prevalence of unsuspected pancreatic cysts on MDCT. Am J Roentgenol 2008;191:802.

3 Dumonceau J-M, Polkowski M, Larghi A, et al. Indications, results, and clinical impact of endoscopic ultrasound (EUS)-guided sampling in gastroenterology: European Society of Gastrointestinal Endoscopy (ESGE) Clinical Guideline. Endoscopy 2011;43:1-16.

4 Sakamoto H, Kitano M, Komaki T, et al. Endoscopic ultrasound-guided neurolysis in pancreatic cancer. Pancreatology 2011;11(Suppl 2):52-8.

5 Dumonceau J-M, Delhaye M, Tringali A, et al. Endoscopic treatment of chronic pancreatitis: European Society of Gastrointestinal Endoscopy (ESGE) Clinical Guideline. Endoscopy 2012;44:784-800.

6 Bakker OJ, van Santvoort HC, van Brunschot S, et al. Endoscopic transgastric vs surgical necrosectomy for infected necrotizing pancreatitis. JAMA 2012;307:1053-61.

7 http://www.bsg.org.uk/clinical/news/ercp-\%E2\%80\% 93-the-way-forward-a-standards-framework.html 